\title{
Resíduos de Serraria no Estado do Pará: Caracterização, Quantificação e Utilização Adequada
}

\author{
Luiz Eduardo de Lima Melo ${ }^{1}$, Cláudia de Jesus Silva², Kamille Vieira Lopes², \\ Pedro Gualberto Martins de Brito ${ }^{2}$, Iêdo Souza Santos ${ }^{3}$ \\ ${ }^{1}$ Programa de Pós-Graduação em Ciência e Tecnologia da Madeira, \\ Departamento de Ciências Florestais, Universidade Federal de Lavras - UFLA \\ ${ }^{2}$ Universidade do Estado do Pará - UEPA \\ ${ }^{3}$ Departamento de Tecnologia da Madeira, Universidade do Estado do Pará - UEPA
}

\begin{abstract}
RESUMO
O objetivo do trabalho foi fazer a caracterização do processo produtivo e dos resíduos gerados por uma serraria localizada na Região Metropolitana de Belém-PA. A caracterização do processo produtivo e a quantificação do volume de resíduos totais gerados foram feitas a partir do balanço de materiais da empresa. Verificou-se que a serraria analisada apresentou percentuais de rendimento significativos, próximos aos valores mais altos encontrados na literatura consultada para serrarias da região. Esse fato pode estar associado ao uso de maquinário de qualidade utilizado no desdobro da madeira ou mesmo à presença de profissionais capacitados. Observou-se também que a serraria analisada destina de forma inteligente seus resíduos, realizando sua comercialização de acordo com maior ou menor valor agregado dos mesmos.
\end{abstract}

Palavras-chave: serraria, resíduo de madeira, processo produtivo.

\section{Sawmill Residues in the State of Para: Characterization, Quantification and Appropriate Use}

\begin{abstract}
The objective of this study was to characterize the production process and the waste generated by a sawmill in the metropolitan region of Belem, State of Para. The production process characterization and the quantification of the total waste volume generated were determined through the analysis of the material balance sheet of the company. It was possible to notice that the analyzed sawmill presented significant income percentage, close to the highest values found in the literature for sawmills in the region, which may be associated to the use of quality machinery in wood processing or even to the presence of trained labor. It was also observed that the studied sawmill intelligently directs its waste, commercializing it in accordance with greater or lesser added value.
\end{abstract}

Keywords: sawmill, wood waste, production process. 
Resíduos industriais florestais são definidos como os subprodutos decorrentes dos desdobros primário e secundário, como também da utilização da madeira. Dessa forma, são resíduos a casca, a costaneira, as pontas, as aparas, as lascas, os nós, o pó de serra e as maravalhas (Numazawa et al., 2003).

A Região Amazônica ainda se destaca no cenário florestal nacional como grande geradora de resíduos provenientes do processamento da madeira. A justificativa para esse fato se encontra tanto na inadequação de uso da matéria-prima, quanto na falta de conhecimento das propriedades tecnológicas básicas da madeira, fato que acarreta, consequentemente, a aplicação de tecnologias inapropriadas para seu processamento.

O conhecimento necessário para a solução desses problemas passa pela caracterização do rendimento produtivo dessas indústrias, dos fatores geradores de resíduos, do volume e do tipo de resíduos existentes e da sazonalidade da geração dos mesmos, além dos possíveis usos que podem ser dados a esse material. Dessa forma, é fundamental a análise de cada fase do processo produtivo, caracterizado pelo balanço de materiais e pela avaliação do rendimento da empresa (Brand et al., 2002).

O objetivo do trabalho foi fazer a caracterização do processo produtivo, a partir do balanço de materiais, de uma serraria localizada no Distrito de Murinin, município de Benevides, Região Metropolitana de Belém-PA, para verificação do rendimento da empresa e percentual de geração de resíduos, fazendo um estudo comparativo com dados bibliográficos de outras serrarias existentes no mesmo Estado.

Foram feitas visitas técnicas a empresa e a partir de informações fornecidas pelo empresário, foi determinado o rendimento do processo produtivo, com base no princípio de balanço de material, que consiste na determinação de entrada (matéria-prima) e saída (produto final), para a qual se empregou a Equação 1.

$V_{\text {residuo }}=V_{\text {entrada }}-V_{\text {saida }}$

em que: $\mathrm{V}_{\text {residuo }}=$ volume de resíduo gerado em $\mathrm{m}^{3} ; \mathrm{V}_{\text {entrada }}=$ volume de madeira bruta que entra no processo $\mathrm{em}^{3} ; \mathrm{V}_{\text {saída }}=$ volume de madeira processada $\mathrm{em}^{3}$.
O cálculo do volume de resíduos gerados foi foi feito com o auxilio da Equação 2.

$N=\frac{V_{\text {saida }}}{V_{\text {entrada }}}$

em que: $\mathrm{N}=$ rendimento em percentagem; $\mathrm{V}_{\text {entrada }}=$ volume de madeira bruta que entra no processo produtivo; $\mathrm{V}_{\text {sáda }}=$ volume de produto acabado.

A determinação do percentual de resíduo gerado na empresa por espécie de madeira processada foi feita em 3 etapas:

- O volume geométrico das toras foi determinado tomando-se o diâmetro médio das extremidades da tora e o comprimento da mesma. Para a determinação do volume de serrados, fez-se a cubagem de cada peça gerada, medindo a espessura, a largura e o comprimento das peças.

- O volume de resíduos foi determinado com base na diferença entre o volume da tora e o volume de serrados obtidos no processamento mecânico, realizando-se a Equação 3.

$V R=V T-V S$

em que: $\mathrm{VR}=$ volume de resíduos em $\mathrm{m}^{3}$; $\mathrm{VT}=$ volume da tora $\mathrm{em}^{3} ; \mathrm{VS}=$ volume de serrados $\mathrm{em}^{3}$.

- A partir do volume total de resíduos calculados por meio da Equação 3, quantificou-se a serragem, aparas e costaneiras com base nos percentuais indicados por Carré \& Schenkel (1992) de 10\% em serragem, por Contente et al. (2002) de 21,54\% em costaneiras e Numazawa et al. (2003) de 21,03\% em aparas de grosso calibre.

$\mathrm{Na}$ Tabela 1, são apresentados os valores fornecidos pelo responsável da serraria no momento da visita, referentes ao volume de entrada de madeira e à saída de produtos por mês, juntamente com os resultados observados para volume de resíduos gerados e rendimento da empresa.

Hillig et al. (2006), estudando o rendimento médio de dez serrarias no Estado do Pará, encontraram grandes diferenças entre estas, sendo o valor mais alto igual a $55 \%$ e o mais baixo igual a $27 \%$. Pode-se constatar que o valor de $50 \%$ de rendimento encontrado para a empresa analisada neste trabalho se encontra bem próximo do valor mais alto de rendimento, determinado pelos autores. 
Na Tabela 2, é apresentado o rendimento de serrados e resíduos gerados e, na Tabela 3 , a repartição de resíduos gerados no processamento primário da tora da espécie de maçaranduba (Manilkara huberi, (Ducke) Chevalier) comercializada pela empresa.

A partir dos resultados encontrados, observouse que a espécie maçaranduba (Manilkara huberi, (Ducke) Chevalier) apresentou um rendimento de serrado superior ao rendimento de $41,2 \%$ encontrado por Dutra et al. (2005), na caracterização de resíduos gerados por uma serraria no município de MojúPA, e bem próximo da média de 50\% encontrada pela SUDAM (1981), em estudo com 32 espécies da Amazônia. Tal fato pode estar relacionado ao nível tecnológico de conversão mecânica da tora em serrado, que, no caso da empresa estudada, se mostrou relativamente superior ao observado nas serrarias tradicionais.

Verificou-se que o maior percentual de resíduos formados se constituiu de aparas, seguidas por costaneiras e serragem. Segundo informações coletadas juntamente ao responsável pela serraria no momento da visita, os resíduos originados no processo produtivo têm sua destinação relacionada ao respectivo valor agregado dos mesmos, indicando por que a lenha e a serragem, sendo de menor valor agregado, são vendidas para fábricas de cerâmicas e de tijolos localizadas no município de Castanhal/PA. Diversamente, as costaneiras e aparas de maior valor agregado são vendidas para pequenas movelarias próximas à serraria. $\mathrm{O}$ destino dado aos resíduos na serraria analisada é semelhante ao proposto por Numazawa et al. (2003): do índice de 42,77\% de aparas, encontrado para a espécie de maçaranduba, pode-se destinar 21,03\%, conforme sugerem, para fabricação de artefatos, correspondendo a $0,14 \mathrm{~m}^{3}$; o restante de $0,54 \mathrm{~m}^{3}$ destina-se para gerar energia, juntamente com a serragem.

Verificou-se que a serraria analisada apresentou percentuais de rendimento significativos, próximos aos valores mais altos encontrados por outros autores em pesquisa com o mesmo enfoque. A serraria apresentou um aproveitamento de serrado em metros cúbicos, para a madeira de maçaranduba, superior aos dados encontrados na literatura, fato que pode estar ligado ao uso de maquinário de qualidade no desdobramento da madeira ou mesmo à presença de profissionais capacitados atuando no processo produtivo da empresa. No que se refere ao aproveitamento dos resíduos madeireiros provenientes do processo, verificou-se que a serraria em questão destina de forma inteligente seus resíduos, realizando sua comercialização de acordo com maior ou menor valor agregado dos mesmos.

Tabela 1. Volume de resíduos gerados e rendimento do processo produtivo com base no balanço de material.

Table 1. Volume of waste generated and yield production process based on material balance.

\begin{tabular}{ccccccc}
\multirow{2}{*}{ Espécie } & \multicolumn{3}{c}{ Volume $\left(\mathbf{m}^{3}\right)$} & & Rendimento serrado & \multicolumn{2}{c}{$\begin{array}{c}\text { Resíduo } \\
\text { (\%) }\end{array}$} \\
\cline { 2 - 4 } Maçaranduba & Tora & Serrado & Resíduo & $(\%)$ & 45 & 55 \\
\hline
\end{tabular}

Tabela 2. Volume total de madeira, serrado e resíduo.

Table 2. Total volume of wood, sawed and residue.

\begin{tabular}{cccc}
$\begin{array}{c}\text { Volume de tora que entra } \\
\text { por } \mathbf{m e ̂ s}\left(\mathbf{m}^{\mathbf{3}}\right)\end{array}$ & $\begin{array}{c}\text { Volume de tora que sai } \\
\text { por } \mathbf{m e ̂ s}\left(\mathbf{m}^{\mathbf{3}}\right)\end{array}$ & $\begin{array}{c}\text { Volume de resíduos } \\
\text { gerados por } \mathbf{m e ̂ s}\left(\mathbf{m}^{3}\right)\end{array}$ & $\begin{array}{c}\text { Rendimento da empresa } \\
(\%)\end{array}$ \\
\hline 1500 & 750 & 750 & 50 \\
\hline
\end{tabular}

Tabela 3. Repartição dos resíduos gerados na serraria.

Table 3. Breakdown of waste generated at the sawmill.

\begin{tabular}{|c|c|c|c|c|c|c|}
\hline \multirow{2}{*}{ Espécies } & \multicolumn{2}{|c|}{ Costaneiras } & \multicolumn{2}{|c|}{ Aparas } & \multicolumn{2}{|c|}{ Serragem } \\
\hline & $\left(\mathrm{m}^{3}\right)$ & $\%$ & $\left(\mathrm{~m}^{3}\right)$ & $\%$ & $\left(\mathrm{~m}^{3}\right)$ & $\%$ \\
\hline Maçaranduba & 0,62 & 39,29 & 0,68 & 42,77 & 0,29 & 18,24 \\
\hline
\end{tabular}




\section{STATUS DA SUBMISSÃO}

Recebido: 19/09/2011

Aceito: 31/01/2012

Resumo publicado online: 08/02/2012

Artigo completo publicado: 31/03/2012

\section{AUTOR(ES) PARA CORRESPONDÊNCIA}

\section{Luiz Eduardo de Lima Melo}

Programa de Pós-Graduação em Ciência e Tecnologia da Madeira,

Departamento de Ciências Florestais, Universidade Federal de Lavras - UFLA, Rua Barbosa Lima, 829, Bloco 01, apto. 304, Centro, CEP 37200-000, Lavras, MG, Brasil e-mail: luizeduardo_47@yahoo.com.br

\section{Cláudia de Jesus Silva}

Universidade do Estado do Pará - UEPA, Rua Nova Cintra, Bairro Curuçamba, Casa 01, Quadra 32, CEP 67145-000, Ananindeua, PA, Brasil e-mail: claudia_jsilva@yahoo.com.br

\section{REFERÊNCIAS}

Brand MA, Muñiz GIB, Silva DA, Umberto K. Caracterização do rendimento e quantificação dos resíduos gerados em serraria através do balanço de materiais. Revista Floresta 2002; 32(2): 247-259.

Carré J, Schenkel. Biomass Characteristics and combustion process. In: Proceedings of the E.C. workshop: Designs and selection of biomass boilers; 1992; Yogojakarta. Yogojakarta; 1992. p. 70.

Contente PLB, Silva JNM. Relação entre volumes de árvores em pé e volume francon. Belém: FCAP-serviço de documentação, 2002. p. 25-41. (FCAP Informe técnico, n. 28).

Dutra RIJP, Nascimento SM, Numazawa S. Resíduos de indústria madeireira: caracterização, conseqüências sobre o meio ambiente e opções de uso. Revista Científica Florestal 2005; 5(2).

Hillig E, Schneider VE, Weber C, Tecchio R. Resíduos de madeira da indústria madeireira - caracterização e aproveitamento. In: Anais do XXVI Encontro Nacional de Engenharia de Produção; 2006; Fortaleza. Associação Brasileira de Engenharia de Produção; 2006. p. 7.

Numazawa S, Carvalho MSP, Brandão ATO, Alves RL, Rodrigues AF. Determinação do índice de conversão da tora em madeira serrada de oito espécies florestais processadas na empresa Comércio Madeira Dunorte Ltda. In: Anais do IX Congresso Internacional de Compensado e madeira Tropical; 2003; Belém. Belém, 2003. p. 22.

Superintendência do Desenvolvimento da Amazônia - SUDAM. Centro de Tecnologia madeireira. Rendimento em serrarias de 30 espécies de madeiras amazônicas. Belém: SUDAM, 1981. 